\title{
ANTIOXIDANT PROPERTIES OF DIHYDROXYFUMARIC ACID AND ITS DIMETHYL ETHER: A COMPARATIVE DFT STUDY OF THEIR REACTIONS WITH THE STABLE RADICAL DPPH*
}

\author{
Mikhail Gorbachev, Natalia Gorinchoy*, Ion Arsene \\ Institute of Chemistry of Academy of Sciences of Moldova, 3, Academiei str., Chisinau MD-2028, Republic of Moldova \\ *e-mail: ngorinchoy@yahoo.com
}

\begin{abstract}
The preferred mechanism of the reaction of dihydroxyfumaric acid and its dimethyl ether with the free stable radical 1,1-diphenyl-2-picrylhydrazyl (DPPH*) was revealed by means of Density Functional Theory (DFT) calculations. The proposed mechanism has an ionic character and includes the formation of charge-transfer complexes as the main stage. It is also shown that the lower antioxidant activity of dimethyl ether of dihydroxyfumaric acid is caused by both its lower acidity (as compared with its precursor acid) and formation of more stable intermediates during its reaction with DPPH*. Our results allow one to rationalize the available experimental data.
\end{abstract}

Keywords: antioxidant activity, DPPH*, dihydroxyfumaric acid, dimethyl ether of dihydroxyfumaric acid, DFT calculations.

Received: September 2014/ Revised final: November 2014/ Accepted: November 2014

\section{Introduction}

Many organic compounds with enol or phenol groups are effective antioxidants which transform various free radicals into their diamagnetic derivatives [1]. Usually their relative antioxidant activity is evaluated by the reaction rate with the free stable radical - DPPH* (1,1-diphenyl-2-picrylhydrazyl) in the methanol-water medium. It was shown [2] that $\mathrm{DPPH}^{*}$ reacts with compounds containing active hydrogen atoms (phenols, mineral and organic acids, etc.) forming 1,1-diphenyl-2-picrylhydrazine. According to [2], DPPH* solutions immediately change their color by adding of diluted aqueous mineral acids therein, in methanol or ethanol (whose acidity is lower) they demonstrate change of the color within several days, while solutions of DPPH* in liquid media without active hydrogen atoms (for instance, in benzene, chloroform, acetone, etc.) remain unchanged within a period of 50 days. Thus, the higher acidity of liquid media leads to the faster decomposition of DPPH*.

In its turn, the rate of the reaction of DPPH* with some phenols depends on the stability of the free radicals formed from the above phenol diamagnetic molecules when their hydrogen atoms migrate to $\mathrm{DPPH}^{*}$ ones. So, for example, isoeugenol reacts very quickly (immediately) with DPPH*, while the reaction between DPPH* and eugenol (whose double bond is not conjugated with its benzene cycle) requires several hours (see [1]). It has been also shown [1] that the compounds containing enol groups (ascorbic acid and isoascorbic one) possess the maximum antioxidant activity, i. e. they are the best scavengers of the free radical DPPH*.

The other effective antioxidant compounds containing in their structures enol groups are the dihydroxyfumaric acid - DHFA and its derivatives (for instance, its dialkylethers). In the work [3] it was shown by means of the method of stopped-flow that in the methanol-water medium the reaction between DHFA and DPPH* includes two stages. During the first stage which requires only $7-10 \mathrm{~s}$ at $\mathrm{T}=293.15 \mathrm{~K}$ one half of $\mathrm{DPPH}^{*}$ reacts with DHFA. The second reaction step is slower and requires around 1-2 hours [3]. Basing on these facts, some theoretical suppositions were done [3] in order to describe the reaction mechanism between DPPH* and DHFA. So, it was assumed that the first stage describes the transfer of one of the two hydroxyl hydrogen atoms of a DHFA molecule to that of DPPH* with formation of 1,1-diphenyl-2-picrylhydrazine. The transfer of the second hydroxyl hydrogen atom of DHFA molecules to the second half of $\mathrm{DPPH}^{*}$ ones has been suggested (see [3]) many times slower.

However, the above-mentioned molecular mechanism is in contradiction with the author's own experimental data [3]. So, the experiment shows that the rate of the fast stage of this reaction is proportional to the square root of the molar concentration of DHFA, whereas in accordance with the assumed mechanism it would have to be proportional to this concentration in the first degree. The given contradiction indicates that the considered mechanism can have an ionic character.

Moreover, in the work [4] the relative antioxidant activity against DPPH* was studied for a number of derivatives of DHFA. It was shown that for each of them the time required for the above two reaction stages is several times longer than that for DHFA. However, these data did not find any theoretical interpretation. As far as we know, there are none quantum-chemical studies clarifying the mechanisms of the reactions of DHFA and its derivatives with DPPH*. Only a number of quantum chemical calculations were mainly devoted to determination of the structure- antioxidant activity relationships for various free radical scavengers $[5,6]$.

In this context the aim of the present work is twofold: to reveal (using DFT calculations) the preferred mechanism of reaction between DHFA and DPPH*; to explain the decrease in the antioxidant activity by passing from DHFA to one of its derivatives, namely, to its dimethyldihydroxyfumarate ether - DMDHFE. 


\section{Computational details}

The electronic and geometrical structures of all the considered compounds have been investigated by means of the DFT method, including Becke's three-parameter nonlocal-exchange functional [7] with the correlation functional of Lee, Yang and Parr (B3LYP) [8]. For each of the compounds considered, a full geometry optimization was carried out using the $6-31 \mathrm{G}^{*}$ basis sets including polarization functions for all the atoms in the systems [9]. The calculations were performed with the GAUSSIAN 09 program package [10].

\section{Results and discussion}

At the first stage of our quantum-chemical calculations we have tried to find the molecular transition state responsible for transfer of the first hydrogen atom from a DHFA molecule to the corresponding first molecule of DPPH*. However, all these attempts have not been successful, that is clear from the chemical point of view. Really, the direct transition of the hydrogen atom from the oxygen atom of DHFA (belonging to one of its hydroxyl groups) to the corresponding nitrogen atom of $\mathrm{DPPH}^{*}$ cannot occur due to the higher electronegativity of the oxygen atom. At the same time the reaction between DPPH* and DHFA is exothermic with the energy gain 0.0075 a.u. or $19.7 \mathrm{~kJ} / \mathrm{mol}$ (see the reaction below, where DHFA is denoted as $\mathrm{AH}_{2}$ in order to show its active hydrogen atoms, and $\mathrm{AH}^{*}$ is the radicalacid formed from $\mathrm{AH}_{2}$ ):

$$
\begin{aligned}
& \mathrm{DPPH}^{*}+\mathrm{AH}_{2} \rightarrow \quad \mathrm{DPPH}-\mathrm{H}+\mathrm{AH}^{*} \\
& -1417.8717 \text { a.u. } \quad-606.1632 \text { a.u. } \quad-1418.5014 \text { a.u. } \quad-605.5411 \text { a.u. } \\
& -2024.0349 \text { a.u. } \quad \text {-2024.0424 a.u. }
\end{aligned}
$$

It means that the above reaction is energetically allowed. This discrepancy shows that the mechanism of the hydrogen transfer from DHFA to DPPH*is essentially more complex. In order to explain it, we have taken into account the above fact that the solutions of DPPH* change their color by adding of mineral acids therein [2]. Based on this circumstance one can assume that the given rapid loss of the characteristic DPPH*solutions can be caused by the fast protonation of this radical.

Basing on this interpretation we have supposed that the reaction between DPPH* and DHFA begins from the protonation of one of the heteroatoms of $\mathrm{DPPH}^{*}$ by means of one of the free protons existing in the water-methanol solution of DHFA due to the electrolytic dissociation of one of its carboxyl groups. In Figure 1 some calculated heteroatoms' charges of DPPH* are presented. The comparison of these charges indicates that the protonation of the $\mathrm{DPPH}^{*}$ molecule will most likely be realized through its oxygen atom O4 (but not by means of the nitrogen atom N2). The such protonation way is more preferable also from the stereochemical point of view. Really, all the oxygen atoms $\mathrm{O} 3, \mathrm{O} 4, \mathrm{O} 5$, and $\mathrm{O} 6$ are out of the plane of the picryl cycle of $\mathrm{DPPH}^{*}$ and, therefore, they shield the nitrogen atom $\mathrm{N} 2$. Besides, the atom N2 is spatially shielded by the bulk phenyl groups connected with N1.

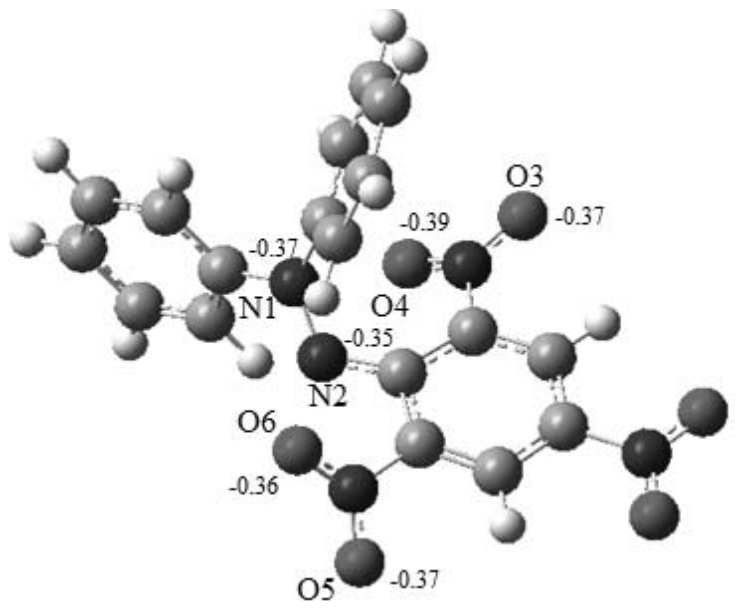

Figure 1. The calculated charges on some heteroatoms in the DPPH* molecule.

Preference for the protonation way via O4 (as compared with that via N2) follows also from the comparison of the calculated total energies of the two arising cation-radical systems: -1418.2152 a.u. and -1418.0283 a.u., respectively.

Note also that calculations of the cation [DPPH-H] ${ }^{*+}$ allow one to understand the above mentioned color changes of DPPH* solutions. Indeed, in the DPPH* molecule the spin density of its unshared electron is mainly localized on the atom N2, while in the above [DPPH-H $]^{*+}$ system it is mainly shared between the atom $\mathrm{O} 3$ and the nitrogen atom connected with $\mathrm{O} 3$ (see Figure 1). It is clear that this shift of the spin density must change the absorption spectrum in the visible region. 
One can expect that the ionic interaction between the cation-radical $[\mathrm{DPPH}-\mathrm{H}]^{*+}$ and the anion of DHFA (hereafter $[\mathrm{AH}]^{-}$) formed due to electrolytic dissociation of the latter will occur without any energy barrier at the room temperature. Our present and the previous [11] quantum-chemical calculations of the whole system ([DPPH-H $]^{*+}$ $\cdots \cdot \mathrm{AH}]^{-}$) indicate the formation of the charge-transfer complex (CTC) presented in Figure 2.

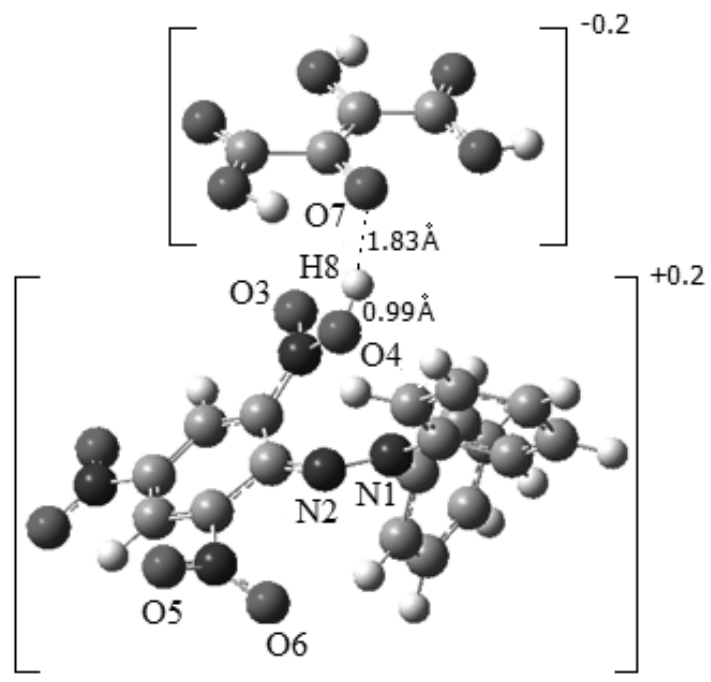

Figure 2. The mutual geometry orientation and the summarized charges in the CTC formed by $[\mathrm{AH}]^{-}$and [DPPH-H $]^{*+}$.

The calculated energy value of the CTC is -2024.0006 a.u. At the same time, the summarized energy of free ions $[\mathrm{DPPH}-\mathrm{H}]^{*+}$ and $[\mathrm{AH}]^{-}$is -2023.8360 a.u. (the energy of $[\mathrm{DPPH}-\mathrm{H}]^{*+}$ is -1418.2152 a.u.; and that of $[\mathrm{AH}]^{-}$is -605.6208 a.u.). Thus, the formation of the CTC from the given ions leads to the energy release of 0.1645 a.u. (i.e. $432.2 \mathrm{~kJ} / \mathrm{mol}$ ). Here it is to be noted (see Figure 2) that the formation of this CTC is accompanied by the transfer of the negative charge $0.8 \mathrm{e}$ from $[\mathrm{AH}]^{-}$to $[\mathrm{DPPH}-\mathrm{H}]^{*+}$. The unshared spin density of the CTC is mainly localized on the carbon atoms belonging to the double $\mathrm{C}=\mathrm{C}$ bond of $[\mathrm{AH}]^{-}$. In its turn, due to the subsequent tautomeric transition of one hydrogen atom from $\mathrm{O} 4$ to $\mathrm{N} 2$ the considered CTC decays into one molecule of 1,1-diphenyl-2-picrylhydrazine and one molecule of the radical-acid $\mathrm{AH}^{*}$. The given decay leads to the energy release which is equal to $97.6 \mathrm{~kJ} / \mathrm{mol}$. Further, two molecules of the radical-acid $\mathrm{AH}^{*}$ easily form their dimmer $(\mathrm{AH})_{2}$ which has four carboxyl groups. The electrolytic dissociation of any of these groups in water-methanol medium gives the anion $\left[\mathrm{A}_{2} \mathrm{H}\right]^{-}$. However, our DFT calculations show that $\left[\mathrm{A}_{2} \mathrm{H}\right]^{-}$is an unstable particle. It decays into one new anion $[\mathrm{AH}]^{-}$of DHFA and one molecule of dioxosuccinic acid [11].

Thus, basing on our DFT calculations, the following mechanism (Scheme 1) can be proposed for the reaction between the acid DHFA $\left(\mathrm{AH}_{2}\right)$ and the free radical DPPH*:

$$
\begin{gathered}
\text { Scheme 1 } \\
\mathrm{AH}_{2} \leftrightarrow[\mathrm{AH}]^{-}+\mathrm{H}^{+} \\
\mathrm{DPPH}^{*}+\mathrm{H}^{+} \rightarrow[\mathrm{DPPH}-\mathrm{H}]^{*+} \\
{[\mathrm{DPPH}-\mathrm{H}]^{*+}+[\mathrm{AH}]^{-} \rightarrow \quad \mathrm{CTC}} \\
\mathrm{CTC} \rightarrow \mathrm{AH}^{*}+1,1 \text {-diphenyl-2-picrylhydrazine } \\
2 \mathrm{HH}^{*} \rightarrow(\mathrm{AH})_{2} \\
(\mathrm{AH})_{2} \stackrel{\leftrightarrow}{\leftrightarrow}\left[\mathrm{A}_{2} \mathrm{H}\right]^{-}+\mathrm{H}^{+} \\
{\left[\mathrm{A}_{2} \mathrm{H}\right]^{-} \rightarrow \stackrel{\mathrm{AH}]^{-}}{\rightarrow}+\text { dioxosuccinic acid }}
\end{gathered}
$$

First of all, it is to be noted here that the mechanism described by Scheme 1 has an ionic character. All its elementary stages (reactions) are accompanied by the corresponding energy release (see the data above). Both these facts allow one to understand the high reaction rate which describes the interaction of DHFA $\left(\mathrm{AH}_{2}\right)$ with the first molecule of $\mathrm{DPPH}^{*}$ (the reaction time is 7-10 s [3]). Moreover, as the volume concentration of $\mathrm{H}^{+}$formed by the reaction (1) is proportional to the square root $\left[\mathrm{AH}_{2}\right]^{1 / 2}$ (due to the law of mass action), so the rate of the reaction (2) which defines the total rate of the interaction between $\mathrm{AH}_{2}$ and the first molecule of $\mathrm{DPPH}^{*}$ will be proportional to the product of the concentrations $\left[\mathrm{DPPH}^{*}\right]\left[\mathrm{H}^{+}\right]$, i.e. $\left[\mathrm{DPPH}^{*}\right]\left[\mathrm{AH}_{2}\right]^{1 / 2}$. Indeed, this theoretical conclusion is completely confirmed by the experimental data [3]. 
In their turn, the new ions $[\mathrm{AH}]^{-}$and $\mathrm{H}^{+}$are formed by the reactions (4)-(7). These new ions will interact with the second molecule of DPPH* (see the stages (2) and (3)). However, their formation is caused by the reaction of the electrolytic dissociation of $(\mathrm{AH})_{2}$ (the dimmer of the radical-acid $\left.\mathrm{AH}^{*}\right)$ and by the further decay of the anion $\left[\mathrm{A}_{2} \mathrm{H}^{-}(\mathrm{see}\right.$ Scheme 1). The realization of these reactions (6) and (7) leads to the corresponding elongation of the time required for the reaction of $\mathrm{AH}_{2}$ with the second molecule of $\mathrm{DPPH}^{*}$ (up to 90 minutes [3]). Thus, the mechanism of the reaction between DPPH* and DHFA found theoretically in the present work allows one to understand these experimental data.

In order to explain the lower antioxidant activity of the derivatives of DHFA (compared to the free acid), we have considered the interaction of dimethyldihydroxyfumarate ether (DMDHFE) with DPPH*. It has been noted in the Introduction that the average reaction time of this process is several times greater than that for the case of DHFA. As above, the DFT calculations do not demonstrate any molecular transition state for the reaction between DPPH* and DMDHFE. In its turn, the described ionic mechanism requires the presence of free protons in reaction medium. In this case the given protons can appear only due to dissociation of one of two hydroxyl groups of DMDHFE. However, these hydroxyl groups dissociate harder than carboxyl ones do [12]. Due to this fact the time required for the elementary reaction (1) in Scheme 1 for DMDHFE will be longer than that for DHFA.

For DMDHFE the elementary reactions (2)-(5) are just the same as in the case of DHFA. So, for example, the optimized geometry of the CTC formed by [DPPH-H] $]^{*+}$ and [DMDHFE] $]^{-}$(where [DMDHFE] $]^{-}$is the anion of DMDHFE) has the same interatomic distances O7-H8 as in the CTC of DHFA (Figure 3).

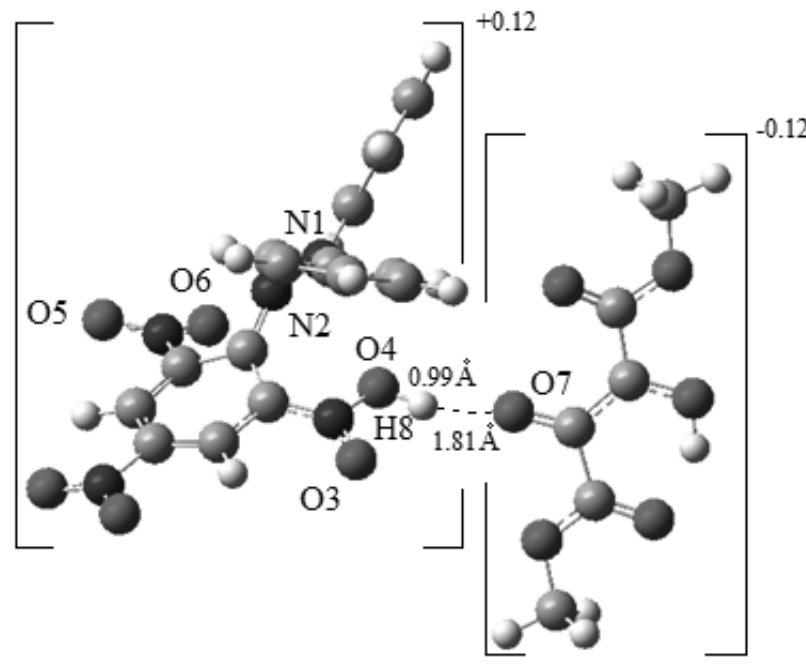

Figure 3. The mutual geometry orientation and the summarized charges in the CTC formed by [DMDHFE $]^{-}$and [DPPH-H] ${ }^{*+}$.

Here it is to be noted the following circumstance. The summarized reaction of the hydrogen transfer between $\mathrm{DPPH}^{*}$ and DHFA $\left(\mathrm{AH}_{2}\right)$ is exothermic with the energy effect of $19.7 \mathrm{~kJ} / \mathrm{mol}$ (see above). However, the similar summarized reaction for DMDHFE:

$$
[\mathrm{DPPH}]^{*}+\mathrm{DMDHFE} \rightarrow[\mathrm{DPPH}-\mathrm{H}]+[\mathrm{DMDHFE}]^{*}
$$

is endothermic. Our DFT calculations show that it requires $20.5 \mathrm{~kJ} / \mathrm{mol}$. At the same time, the reaction of the dimerization of the radicals [DMDHFE]*:

$$
2[\mathrm{DMDHFE}]^{*} \rightarrow[\mathrm{DMDHFE}]_{2}
$$

is an exothermic process. It is accompanied with the energy release of $96.0 \mathrm{~kJ} / \mathrm{mol}$. This energy is sufficient for realization of the above reaction between DPPH* and DMDHFE (when the considered molecule of DMDHFE interacts with its first molecule of DPPH*). Thus, the summarized process:

$$
2[\mathrm{DPPH}]^{*}+2 \text { DMDHFE } \rightarrow 2[\mathrm{DPPH}-\mathrm{H}]+[\mathrm{DMDHFE}]_{2}
$$

will be also exothermic as in the case of DHFA.

It is to be noted that the dimmer [DMDHFE] $]_{2}$ is formed (similar to the reaction (5) in Scheme 1) by two radicals [DMDHFE]* received in its turn after the decay of the CTC between [DPPH-H] ${ }^{*+}$ and [DMDHFE] $]^{-}$. However, our calculations show that $[\mathrm{DMDHFE}]_{2}$ is not the end product of the reaction between $\mathrm{DPPH}^{*}$ and DMDHFE. The optimized structure of the dimmer [DMDHFE] $]_{2}$ is shown in Figure 4. 


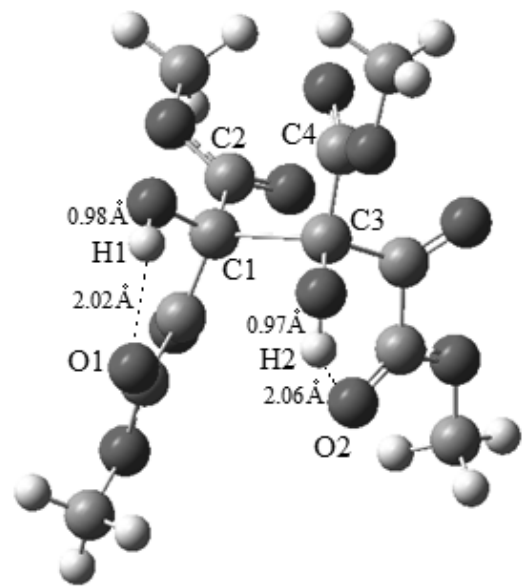

Figure 4. The optimized structure of $[\mathrm{DMDHFE}]_{2}$ with the intramolecular hydrogen bonds.

It contains the intramolecular hydrogen bonds (marked by the dotted lines) which prevent the possible dissociation of any of the hydroxyl groups of [DMDHFE $]_{2}$. In this case the ionization of one of its hydroxyl groups (due to elimination of the corresponding proton) does not lead to the further decay of the anion $\left[[\mathrm{DMDHFE}]_{2}\right]^{-}$as it takes place in the considered case of DHFA (See the reactions (6) and (7) in Scheme 1, and the work [12]). Further, our calculations indicate that the new radical $\left[[\mathrm{DMDHFE}]_{2}\right]^{*}$ formed by the reaction between $\left[[\mathrm{DMDHFE}]_{2}\right]^{-}$and the cation-radical $[\mathrm{DPPH}-\mathrm{H}]^{*+}$ is also a relatively stable particle. Its molecular structure demonstrates only one elongated (up to $1.63 \AA$ ) bond $\mathrm{C} 1-\mathrm{C} 2$ which is shown by the dotted line in Figure 5.

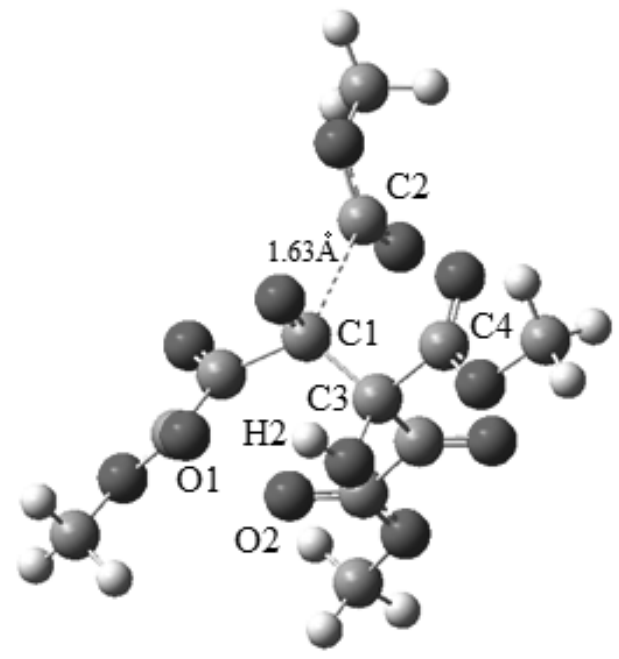

Figure 5. The optimized structure of the radical [[DMDHFE $\left.]_{2}\right] *$.

In its turn, the derivative DV of $\left[[\mathrm{DMDHFE}]_{2}\right]^{*}$ received by the elimination of the last hydrogen atom from the latter (H2 in Figure 5) due to its reaction with the new molecule of $\mathrm{DPPH}^{*}$ (via the above-considered formation of $\left.[\mathrm{DPPH}-\mathrm{H}]^{*+}\right)$ is the essentially more active particle than $\left[[\mathrm{DMDHFE}]_{2}\right]^{*}$. This derivative spontaneously decays into two molecules of dimethyl ether of dioxosuccinic acid. Our calculations show that the following final reactions take place:

$$
\begin{aligned}
& {\left[[\mathrm{DMDHFE}]_{2}\right]^{*}+\mathrm{DPPH}^{*} \rightarrow \text { DPPH-H }+ \text { DV }} \\
& -1367.6810 \text { a.u. } \quad-1417.8717 \text { a.u. } \quad-1418.5014 \text { a.u. } \quad-1366.9674 \text { a.u. } \\
& -2785.5527 \text { a.u. } \\
& -2785.4688 \text { a.u. } \\
& \mathrm{DV} \quad \rightarrow \quad 2 \mathrm{CH}_{3} \mathrm{O}-(\mathrm{C}=\mathrm{O})_{4}-\mathrm{OCH}_{3} \\
& -1366.9674 \text { a.u. } \quad 2 \times(-683.5629 \text { a.u. })=-1367.1258 \text { a.u. }
\end{aligned}
$$

The summarized energy release of the above reactions equals to 0.0745 a.u. $(195.7 \mathrm{~kJ} / \mathrm{mol})$. It means that the transformation of [[DMDHFE $\left.]_{2}\right] *$ into dimethyl ether of dioxosuccinic acid is an exothermic process.

Interaction of the antioxidants DHFA and DMDHFE with DPPH* requires the electrolytic dissociation of their neutral molecules (see the first stage in Scheme 1). It means that the compound with the higher acidity will have the higher antioxidant activity. 
In the case of DHFA one molecule possesses four active hydrogen atoms (two of them belong to its easily dissociated carboxyl groups and two belong to its hydroxyl ones). On the other hand, DMDHFE, whose antioxidant activity is essential lower, has only two hydrogen atoms belonging to two hardly dissociating hydroxyl groups per one molecule. Thus, we can conclude that any increase in molecular acidity of an antioxidant will lead to the corresponding increase in its antioxidant activity (against DPPH*). The same conclusion holds not only for DHFA and DMDHFE [3, 4], but also for other antioxidants. For example, the antioxidant activity of various derivatives of gossypol against DPPH* increases, firstly, with the increase in the number of hydroxyl phenol groups per one molecule (i.e. with the increase in the number of active acid hydrogen atoms) and, secondly, by passing from neutral compounds to their derivatives containing positively charged organic cations whose phenol hydroxyl groups possess the increased acidity [13].

It is clear that the consecutive formation of $[\mathrm{DMDHFE}]_{2}$, $\left[[\mathrm{DMDHFE}]_{2}\right]^{-}$, and $\left[[\mathrm{DMDHFE}]_{2}\right]^{*}$ together with the just considered decay of [[DMDHFE $\left.]_{2}\right]^{*}$ (via DV) require an essential additional time for their realization. The given circumstances, along with the above-mentioned low acidity of hydroxyl groups of DMDHFE, allow one to understand the sufficient decrease in the rate of the reaction between DPPH* and DMDHFE [4].

\section{Conclusions}

The following conclusions can be drawn. The preferred mechanisms describing the antioxidant activities of dihydroxyfumaric acid and its dimethyl ether were proposed on the basis of the DFT calculations of their reactions with the stable radical DPPH*. It is shown that the main stage of both these reactions is the formation of the charge-transfer complexes. They are formed from the protonated $\mathrm{DPPH}^{*}$ molecules and the anions of the above antioxidants arising due to their electrolytic dissociation in the water-methanol medium. The comparison of these two cases indicates that the studied antioxidant activity increases together with the acidity of the investigated compounds, i.e. by passing from the dimethyl ether to its precursor acid. The lower antioxidant activity of the ether is also caused by formation of additional number of intermediates during its reaction with $\mathrm{DPPH}^{*}$. The found results are in a good agreement with the available experimental data. In particular, they allow one to explain the kinetic data describing the experimental concentration dependences for the rates of the investigated reactions and to understand the high stability of DPPH* solutions in various solvents without active hydrogen atoms.

\section{References}

1. Brand-Williams, W.; Cuvelier, M.E.; Berset, C. Use of a Free Radical Method to Evaluate Antioxidant Activity. LWT - Food Science and Technology, 1995, 28, pp. 25-30.

2. Yordanov, N.D. Is Our Knowledge about the Chemical and Physical Properties of DPPH Enough to Consider It as a Primary Standard for Quantitative EPR Spectrometry. Applied Magnetic Resonance, 1996, 10, pp. 339-350.

3. Secara, N. Stopped-Flow Spectrophotometric Studies of the Kinetics of Interaction of Dihydroxyfumaric Acid with the DPPH Free Radical. Chemistry Journal of Moldova, 2010, 5(2), pp. 83-87.

4. Secara, N.; Duca, Gh.; Vlad, L.; Macaev, F. Observation on the Antioxidant Activity of Novel Dixydroxyfumaric Acis Derivatives. Chemistry Journal of Moldova, 2010, 5(2), pp. 59-67.

5. Borges, R.S.; Queiroz, A.N.; Mendes, A.P.S.; Araújo, S.C.; França, L.C.S.; Franco, E.C.S.; Leal, W.G.; da Silva, A.B.F. Density Functional Theory (DFT) Study of Edaravone Derivatives as Antioxidants. International Journal of Molecular Sciences, 2012, 13, pp. 7594-7606.

6. Velkov, Z.A.; Kolev, M.K.; Tadjer, A.V. Modeling and statistical analysis of DPPH scavenging activity of phenolics. Collection of Czechoslovak Chemical Communications, 2007, 72, pp.461-1471.

7. Kohn, W.; Becke, A.D.; Parr, R.G. Density functional theory of electronic structure. Journal of Physical Chemistry, 1996, 100, pp. 12974-12980.

8. Lee, C.; Yang, W.; Parr, R.G. Development of the Colle-Salvetti correlation-energy formula into a functional of the electron density. Physical Review B, 1988, 37, pp. 785-789.

9. Hehre, W.J.; Radom, L.; Schleyer, P.V.R.; Pople, J.A. Ab Initio Molecular Orbital Theory; Wiley: New York, 1986, $548 \mathrm{p}$.

10. Frisch, M. J.; Trucks, G.W.; Schlegel, H.B. et al. Gaussian 09, Revision B.01; Gaussian, Inc.: Wallingford, CT, 2009.

11. Gorbachev, M.Yu.; Gorinchoy, N.N. DFT Evidence of Ionic Character of Interaction of Dihydroxyfumaric Acid with the Free Radical DPPH. In Conference book of $24^{\text {th }}$ Austin Symposium on Molecular Structure and Dynamics, Dallas, USA, March 3-6, 2012, p. 159.

12. Gorbachev, M.Yu.; Gorinchoy, N.N.; Arsene, I. Comparative DFT Study of Interaction between Dimethyldihydroxyfumarate and its Precursor Acid with the Stable Radical DPPH*. In Book of Abstracts of the $\mathrm{XVII}^{\text {th }}$ International Conference "Physical Methods in Coordination and Supramolecular Chemistry", Chisinau, Republic of Moldova, October 24-26, 2012, p. 34.

13. Ilkevich, N.S.; Rybachenko, V.I.; Shroeder, G.; Dmitruk, A.F.; Chotyi, K.Yu. Antioxidative properties of gossypol and some its imino-derivatives. Proceedings of Donetsk National Technical University, Series: Chemistry and Chemical Engineering, 2009, Section 2, pp. 110-117 (in Russian). 